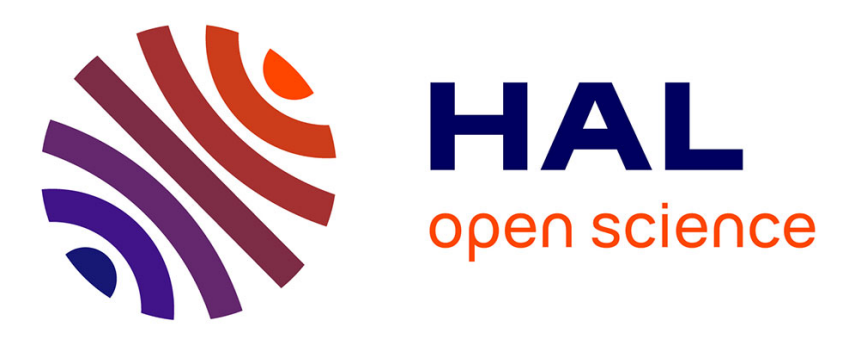

\title{
Simulation of spatially distributed intensive biological systems
}

Antoine Haddon, Victor Alcaraz-Gonzalez, Maha Hmissi, Jérôme Harmand, Antoine Rousseau

\section{- To cite this version:}

Antoine Haddon, Victor Alcaraz-Gonzalez, Maha Hmissi, Jérôme Harmand, Antoine Rousseau. Simulation of spatially distributed intensive biological systems. ECC 2020 - European Control Conference, May 2020, Saint Pettersburg, Russia. pp.575-580, 10.23919/ECC51009.2020.9143773 . hal$02480178 \mathrm{v} 2$

\section{HAL Id: hal-02480178 \\ https://hal.inria.fr/hal-02480178v2}

Submitted on 17 Feb 2020

HAL is a multi-disciplinary open access archive for the deposit and dissemination of scientific research documents, whether they are published or not. The documents may come from teaching and research institutions in France or abroad, or from public or private research centers.
L'archive ouverte pluridisciplinaire HAL, est destinée au dépôt et à la diffusion de documents scientifiques de niveau recherche, publiés ou non, émanant des établissements d'enseignement et de recherche français ou étrangers, des laboratoires publics ou privés. 


\title{
Simulation of spatially distributed intensive biological systems
}

\author{
Antoine Haddon ${ }^{1,2}$, Victor Alcaraz-Gonzalez ${ }^{3}$, Maha Hmissi ${ }^{4}$, Jérôme Harmand ${ }^{5}$ and Antoine Rousseau ${ }^{6}$
}

\begin{abstract}
We introduce a new type of bioreactor model that offers a representation of the spatial features of a pilot scale upflow fixed bed reactor. This model couples fluid dynamics and biological activity in order to obtain the internal gradient of substrate and biomass concentrations. We take advantage of reactor geometry to reduce the model to a single spatial dimension in the section containing the fixed bed but in other sections, we consider a 3D model with Navier-Stokes equations for the fluid dynamics. To represent the biological activity, we use a 2 step model and for the substrates, advectiondiffusion-reaction equations. We only consider the biomasses that are attached in the fixed bed section and to take into account crowding effects, we model their growth with a density dependent function. We show that this model can reproduce the spatial gradient of experimental data and helps to better understand the internal dynamics of the reactor.
\end{abstract}

\section{INTRODUCTION}

Mathematical modelling of bioreactors has been recognized as an important tool for the analysis, control and optimization of bioprocesses. In addition to giving a deeper insight into the process, modelling allows to evaluate different bioreactor designs or operational scenarios. Models have been developed that are capable of representing spatial variations of reactor contents and these differ from classical models that focus on the biochemical kinetics and generally assume that the reactor is perfectly mixed.

The first examples of such models, known as compartment models, represent a reactor as a network of interconnected well-mixed zones and thus use systems of ordinary differential equations (ODE), which facilitates analysis and simulation [1], [2], [3].

The emergence of computer fluid dynamics (CFD) has allowed the simulation of bioreactor models that take into account complex physical processes. At first, only fluid dynamics were considered [4], [5], but recent studies also include bio-reactions, leading to even more complex models [6], [7], [8].

A third type of model attempts to find a compromise between model complexity and physical accuracy. These models represent a reactor in 1 or 2 spatial dimensions with

\footnotetext{
${ }^{1}$ Mathematical Engineering Department and Center for Mathematical Modelling (CNRS UMI 2807), Universidad de Chile, Santiago, Chile. ahaddon@dim.uchile.cl

${ }^{2}$ MISTEA, Univ. Montpellier, INRA, Montpellier SupAgro, Montpellier, France.

${ }^{3}$ Departamento de Ingeniería Química, Universidad de Guadalajara, Guadalajara, México.

${ }^{4}$ Université de Tunis El Manar, École Nationale dIngénieurs de Tunis, Laboratoire de modélisation en Hydraulique et Environnement, Tunis Le Belvédère, Tunisia.

${ }^{5}$ LBE, INRA, Narbone, France.

${ }^{6}$ Inria, IMAG, Univ. Montpellier, CNRS, Montpellier, France.
}

simple fluid dynamics or even assuming that fluid velocity is constant in space to focus on bioreactions [9], [10]. The advantage of reducing complexity is that it allows a more indepth analysis or the consideration of optimization problems [11], [12], [13], [14].

The objective of this work is to present a new type of model that offers a representation of spatial features more accurate than reduced complexity models but that is still tractable for optimization of bioreactor design and operation. For this, we represent reactor geometry precisely but take advantage of special features to obtain a mixed 3D - 1D model.

We model here a pilot scale reactor operated in Guadalajara-Jalisco (Mexico) that is used to treat diluted tequila vinasses by anaerobic digestion and produce biogas. A spatial gradient was observed during experiments and data was gathered by collecting substrate and byproducts at different points along the main axis of the reactor. This was used to develop a compartment model consisting of two interconnected homogeneous zones [15]. This model was able to reproduce the spatial gradient roughly and the present work aims at improving the representation of spatial features.

In Section II, we present the model and, in Section III, the challenges of its numerical simulation. Section IV deals with parameter estimation and model verification.

\section{MODEL}

The bioreactor that we model here is a vertical cylinder with the influent to be treated entering at the bottom and exiting at the top (Fig. 1). A fraction of the liquid from the top is recirculated and mixed with the influent before re-entering the tank at the bottom. Mixing is also helped by pumping out liquid from the very bottom of the tank which is injected back into the tank at the same height. Furthermore, both the inflows at the bottom are such that the liquid enters nearly tangentially in order to induce a circular current in the bottom of the tank. In the middle, there is a fixed bed made of vertical PVC tubes with honeycomb structures (Cloisonyl $($ ), which helps to fix the biomass.

The configuration of this reactor, and in particular the arrangement of the input and output flows, means that we need to consider a 3D model and can not use symmetry to reduce the spatial dimensions for the lower and upper parts of the reactor. However, the PVC tubes in the middle section of the reactor are narrow and once the fluid has entered one of these tubes from the bottom, it remains in the same tube until it reaches the top section. We can therefore consider that the contents inside each tube are homogeneous in the radial directions and only model the spatial distribution 


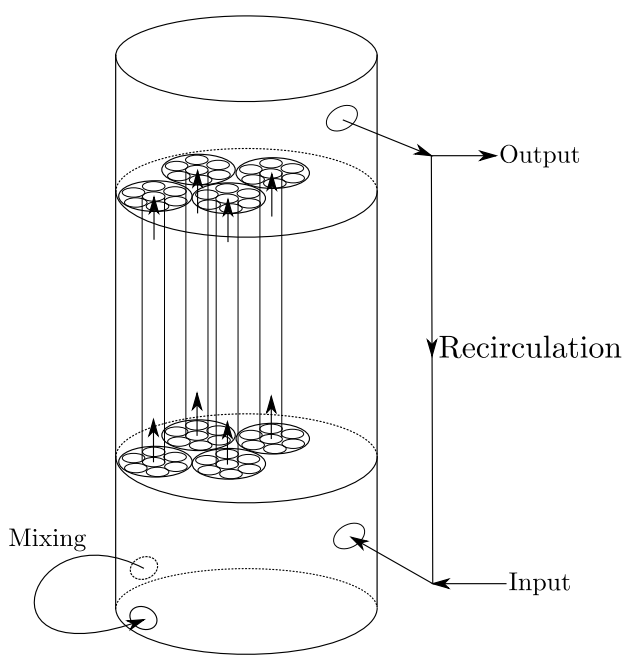

Fig. 1. Schematic view of the reactor

of substances along the vertical dimension. Furthermore, observations of this reactor have reported that most of the active biomass is attached to the walls of the tubes and we will thus neglect the suspended biomass, considering that the bio-reactions take place only in the middle section.

The result is a model of the reactor in 3 parts : bottom and top sections in 3D, where we must consider the fluid dynamics to compute the spatial distribution of the substrates; and the middle section, modelled as an array of parallel 1D tubular reactors, thereby reducing considerably the complexity of the model in this section.

\section{A. Fluid Dynamics}

The vinasses that are treated are completely liquid and do not contain any solid material, so that we can consider that the contents of the reactor are only in liquid or gas phases. We will model the liquid phase as an incompressible viscous fluid, having similar physical properties to water. It is important to note that the timescale of fluid dynamics is much shorter than the biological timescales and therefore we can consider that the liquid flow remains in a steady state, as long as the input-output flows are constant.

The gases produced by the biological activity collect at the top of the reactor and the main impact that gases can have on reactor dynamics is through pressure. Indeed, the solubility and other physico-chemical properties of certain gases can vary with pressure, such as $\mathrm{CO}_{2}$ for instance. This effect could be taken into account by adding a boundary condition for the pressure at liquid-gas interface at the top of the reactor, with the pressure of the gas phase obtained from the ideal gas law. However, we are primarily interested in the biological activity and we can estimate biogas production through the methane generation. Indeed, due to the very low solubility of methane, its molar flow rate is unaffected by pressure. Therefore, we will neglect the dynamics of gases and consider that the upper boundary of the liquid is fixed and similar to a wall.

We therefore use the 3D steady state incompressible
Navier Stokes equations to compute the fluid velocity and pressure in the bottom and top sections. Denoting the fluid velocity $U=\left(u_{x}, u_{y}, u_{z}\right), p$ the pressure, $g$ the acceleration due to gravity and $\nu$ the dynamic viscosity,

$$
\begin{aligned}
U \cdot \nabla U-\nu \Delta U+\nabla p & =g, \\
\nabla \cdot U & =0 .
\end{aligned}
$$

Concerning boundary conditions, for the walls of the bioreactor we consider a no-slip condition, $U=0$. For the inflow and the mixing system, we consider classical Poiseuille flow profiles: $U_{B i n}, U_{m i}, U_{m o}, U_{U o}$ are unitary parabolic functions, $\alpha$ is the angle between the direction of injection and the $n$ is outwards unit normal, the boundary conditions are then

$$
\begin{array}{ll}
U=Q_{t o t}\left(U_{B i n} \cos \alpha, U_{B i n} \sin \alpha, 0\right) & \text { (inflow) } \\
U=Q_{m}\left(U_{m i} \cos \alpha, U_{m i} \sin \alpha, 0\right) & \text { (mixing in) } \\
U=Q_{m} U_{m o} n & \text { (mixing out) } \\
U=Q_{t o t} U_{U o} n & \text { (outflow) }
\end{array}
$$

We denote the total input flow rate as $Q_{t o t}=Q_{r}+Q_{i n}$, the influent flow rate $Q_{i n}$, the re-circulation flow rate $Q_{r}$ and the mixing flow rate $Q_{m}$.

For the interface between the bottom and middle and between the middle and top, where the vertical inner tubes begin and end, we will neglect a small interface layer and consider that the flow is completely in the vertical direction. Denoting $A$ the sectional surface area, we have the following boundary condition for the interfaces

$$
u_{x}=0, \quad u_{y}=0, \quad u_{z}=\frac{Q_{t o t}}{A} .
$$

Similarly, in the middle section, as we model the tubes as 1D vertical tubular bioreactors, we also suppose that the fluid velocity is constant and equal to the velocity at the interfaces.

\section{B. Bio-Kinetics}

For the anaerobic digestion process, we consider the two reaction model of [16]. The first reaction, acidogenesis, represents the degradation of organic matter $S_{1}$ into volatile fatty acids (VFA) $S_{2}$ by acidogenic microorganisms $B_{1}$. Then methanogenic archea $B_{2}$ transform the VFA into methane,

$$
k_{1} S_{1} \stackrel{r_{1}}{\longrightarrow} B_{1}+k_{2} S_{2}, \quad k_{3} S_{2} \stackrel{r_{2}}{\longrightarrow} B_{2}+k_{4} \mathrm{CH}_{4} .
$$

Here $r_{1}, r_{2}$ are the reaction rates and $k_{1}, k_{2}, k_{3}, k_{4}$ are the yield coefficients. In [16] the reaction rate were taken

TABLE I

PHYSICAL PARAMETERS

\begin{tabular}{clcc}
\hline Parameter & & Value & Unit \\
\hline $\mathrm{g}$ & Acceleration due to gravity & 9.81 & $\mathrm{~m}^{2} \mathrm{~s}^{-1}$ \\
$\nu$ & Fluid viscosity & $10^{-3}$ & $\mathrm{~m}^{2} \mathrm{~s}^{-1}$ \\
$\mathrm{D}_{s}$ & Substrate diffusion coefficient & $10^{-7}$ & $\mathrm{~m}^{2} \mathrm{~s}^{-1}$ \\
$Q_{i n}$ & Influent flow rate & 5 & $\mathrm{~L} \mathrm{~h}^{-1}$ \\
$Q_{r}$ & Recirculation flow rate & 150 & $\mathrm{~L} \mathrm{~h}^{-1}$ \\
$Q_{m}$ & Mixing flow rate & 1000 & $\mathrm{~L} \mathrm{~h}^{-1}$ \\
\hline
\end{tabular}


TABLE II

BIOLOGICAL PARAMETERS

\begin{tabular}{clcc}
\hline Parameter & & Value & Unit \\
\hline$S_{1}^{i f}$ & Influent COD concentration & 6.5 & $\mathrm{~g} \mathrm{~L}^{-1}$ \\
$S_{2}^{i f}$ & Influent VFA concentration & 100 & $\mathrm{mmol} \mathrm{L}^{-1}$ \\
$\mu_{1}^{\max }$ & Maximum biomass growth rate (Acidogenesis) & 1.96 & $\mathrm{~d}^{-1}$ \\
$\mu_{2}^{\max }$ & Maximum biomass growth rate (Methanogenesis) & 1.14 & $\mathrm{~d}^{-1}$ \\
$K_{1}^{s}$ & Half saturation constant (Acidogenesis) & 8.16 & $\mathrm{~g} \mathrm{~L}^{-1}$ \\
$K_{2}^{s}$ & Half saturation constant (Methanoogenesis) & 14.44 & $\mathrm{mmol} \mathrm{L}^{-1}$ \\
$K_{i}$ & Substrate inhibition constant (Methanogenesis) & 416.77 & $\mathrm{mmol} \mathrm{L}^{-1}$ \\
$k_{1}$ & Yield for COD degradation & 28.35 & - \\
$k_{2}$ & Yield for VFA production & 186.45 & - \\
$k_{3}$ & Yield for VFA consumption & 58.25 & - \\
$k_{4}$ & Yield for CH 4 production (Methanogenesis) & 453 & - \\
$\tau_{1}$ & Biomass death rate (Acidogenesis) & $0.1 \mu_{1}^{\max }$ & $\mathrm{d}^{-1}$ \\
$\tau_{2}$ & Biomass death rate (Methanogenesis) & $0.1 \mu_{2}^{\max }$ & $\mathrm{d}^{-1}$ \\
$c_{1}^{i}$ & Biomass inhibition constant (Acidogenesis) & 0.6 & $\left(\mathrm{~g} \mathrm{~L}^{-1}\right)^{1 / 2}$ \\
$c_{2}^{i}$ & Biomass inhibition constant (Methanogenesis) & 0.8 & $\left(\mathrm{~g} \mathrm{~L}^{-1}\right)^{1 / 2}$ \\
\hline
\end{tabular}

as $r_{k}=\mu_{k}\left(S_{k}\right) B_{k}$, for $k=1,2$, where the specific growth rates $\mu_{1}, \mu_{2}$ are the Monod and Haldane growth functions

$$
\mu_{1}(s)=\frac{\mu_{1}^{\max } s}{K_{1}^{s}+s}, \quad \mu_{2}(s)=\frac{\mu_{2}^{\max } s}{K_{2}^{s}+s+s^{2} / K_{i}} .
$$

However in this work, in order to take into account the effects of crowding and the fact that a majority of the biomass is fixed to the walls of the tubes in the middle section, we will consider density dependent growth rates $r_{k}=$ $\mu_{k}\left(S_{k}\right) g_{k}\left(B_{k}\right) B_{k}$. Since we model the tubes in 1 dimension, we consider that at every point of these tubes there is a two dimensional colony of microorganisms attached to the wall of the tube with the substrate passing through the center. Biomass inhibition functions have been proposed, for a two dimensional colony of microorganisms in [17] and we will consider the following function

$$
g_{k}(B)=\frac{1}{1+c_{k}^{i} \sqrt{B_{k}}} .
$$

We now detail the model equation for the substrate and biomass concentrations in the bottom, middle and top sections.

a) Bottom and Top: We suppose that there is no biomass in these sections of the reactor and therefore no reaction. The evolution of the substrate concentration $S_{k}$, for $k=1,2$, is modelled by the following advection-diffusion equation

$$
\partial_{t} S_{k}+U \cdot \nabla S_{k}-D_{s} \Delta S_{k}=0
$$

where $D_{s}$ is the diffusivity of the substrates. The boundary conditions for the walls and the outflow are

$$
\nabla S_{k} \cdot n=0
$$

For the inflow, we consider flux equality conditions

$$
\left(S_{k} U+D_{s} \nabla S_{k}\right) \cdot n=S_{k}^{i n} U \cdot n
$$

Here $S_{k}^{i n}$ is the substrate inflow concentration,

$$
S_{k}^{i n}=\frac{Q_{r} S_{k}^{r}+Q_{i n} S_{k}^{i f}}{Q_{r}+Q_{i n}}
$$

with $S_{k}^{i f}$ the influent concentration and $S_{k}^{r}$ the substrate recirculation concentration coming from the upper section of the bioreactor which is computed as

$$
S_{k}^{r}(t)=\frac{1}{\left|\Gamma_{o}\right|} \int_{\Gamma_{o}} S_{k} d \sigma
$$

denoting $\Gamma_{o}$ the part of the boundary where the liquid leaves the reactor. For the auxiliary mixing system, we take similar boundary conditions

$$
\begin{aligned}
\left(S_{k} U+D_{s} \nabla S_{k}\right) \cdot n & =S_{k}^{\text {mix }} U \cdot n & & \text { (mixing in) } \\
\nabla S_{k} \cdot n & =0 & & \text { (mixing out) }
\end{aligned}
$$

with, as before, $\Gamma_{m o}$ denoting the part of the boundary where the liquid is pumped into the mixing system,

$$
S_{k}^{\text {mix }}(t)=\frac{1}{\left|\Gamma_{m o}\right|} \int_{\Gamma_{m o}} S_{k} d \sigma .
$$

At the interface between the bottom and middle section, we could consider a flux equality condition,

$$
\left.\left(S_{k} U+D_{s} \nabla S_{k}\right) \cdot n\right|_{\Omega_{B}}=\left.\left(S_{k} U+D_{s} \nabla S_{k}\right) \cdot n\right|_{\Omega_{M}}
$$

where $\Omega_{B}$ is the bottom and $\Omega_{M}$ is the middle section. However, it is well known that for advection-diffusion equations, when the diffusion coefficient is small, this type of interface conditions can be well approximated by using the following artificial transparent boundary condition [18],

$$
\nabla S_{k} \cdot n=0 .
$$

Finally, for the boundary condition for the top section, corresponding to the middle-top interface, the substrate concentrations are obtained by interpolating the values of the top of the tubes.

b) Middle: This is the section of the bioreactor with the honeycomb structure; there are large tubes each divided in smaller tubes, and we model each one as a 1D vertical tubular bioreactor.

The substrate concentrations, $S_{k}$ for $K=1,2$, satisfy the following equation, in each tube,

$$
\partial_{t} S_{k}+u_{z} \partial_{z} S_{k}-D_{s} \partial_{z z}^{2} S_{k}=f_{k}(\xi),
$$



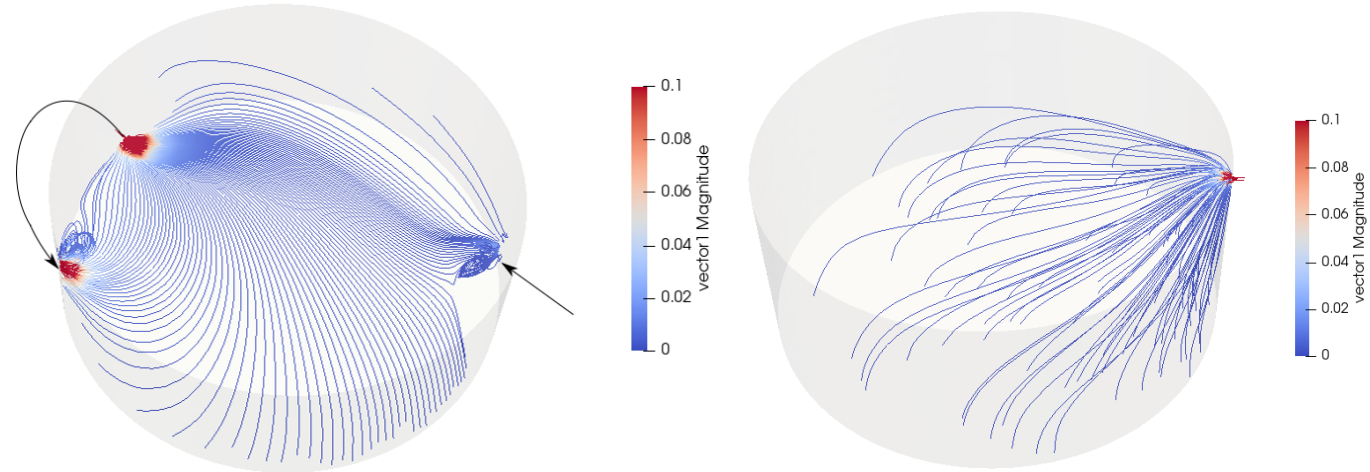

Fig. 2. Streamlines of fluid flow, (left) bottom section with inflow on the right and mixing system on the left and (right) top section.

where $f_{k}(\xi)$ corresponds to the biological reaction with $\xi=$ $\left(S_{1}, S_{2}, B_{1}, B_{2}\right)$,

$$
\begin{aligned}
& f_{1}(\xi)=-k_{1} \mu_{1}\left(S_{1}\right) g_{1}\left(B_{1}\right) B_{1} \\
& f_{2}(\xi)=k_{2} \mu_{1}\left(S_{1}\right) g_{1}\left(B_{1}\right) B_{1}-k_{3} \mu_{2}\left(S_{2}\right) g_{2}\left(B_{2}\right) C_{2} .
\end{aligned}
$$

For boundary conditions, for each tube, at the bottom interface we consider a Dirichlet condition for which we take the average value over the tube area of the corresponding substrate concentration from the bottom section. For the top interface, we take again an artificial transparent boundary

$$
\frac{\partial S_{k}}{\partial z}=0 .
$$

We suppose that the micro-organisms are fixed to the wall of the tubes so that the biomass concentrations are neither advected nor diffused, but we take into account their death rate $\tau_{k}$

$$
\partial_{t} B_{k}=\mu_{k}\left(S_{k}\right) g_{k}\left(B_{k}\right) B_{k}-\tau_{k} B_{k},
$$

Note that this is a family of ordinary differential equations, so that no boundary conditions are required.

For the methane flow rate, as proposed in [16], we will consider that it is proportional to the growth rate of the second biomass. To compute the total flow rate for the whole reactor, we need to integrate over all the middle section

$$
Q_{C H_{4}}=A \sum_{i=1}^{N_{t}} \int_{z_{0}}^{z_{1}} k_{4} \mu_{2}\left(S_{2}\right) g_{2}\left(B_{2}\right) B_{2} d z .
$$

where $N_{t} \approx 100$ is the total number of tubes and $z_{0}, z_{1}$ denote the bottom and top height of the tubes.

\section{Numerical SOLUTiON}

To solve the model equations we use the Finite Element method implemented in the open source library FreeFem++ [19]. For the fluid dynamics, we solve the time dependent Navier-Stokes equations until reaching a steady state. We consider the well established combination of a characteristics method, to deal with the non-linear convection term [20], and P2-P1 type finite elements [21]. Fig. 2 illustrates the computed steady state flow.

For the substrate equations of the bottom and top sections, we also use a finite element method with an implicit time discretization, which presents the advantage of being stable for larger time steps and coarser meshes than an explicit time discretization. However, to correctly solve these equations it is necessary to take time steps of the order of the fluid timescale which is very small compared to the biological timescale. Indeed, especially in the bottom with high fluid velocities due to the mixing system, we need to take time steps of the order of seconds, whereas we are interested in the dynamics of the bioprocess over several days.

Another complication comes from the very small diffusion coefficients of the substrates. For example, a typical component of VFAs, acetic acid is reported to have a diffusion coefficient of the order of $D_{s}=10^{-9} \mathrm{~m}^{2} / \mathrm{s}$ [22]. The consequence is that the substrate concentrations can present sharp spatial variations and thus fine meshes must be used to correctly solve the equations. Then, for smaller diffusion coefficients, the computational cost of simulations is substantially increased, as for example with $D_{s}=10^{-8}$ $\mathrm{m}^{2} / \mathrm{s}$ it is necessary to use a mesh with 4 times more points than for $D_{s}=10^{-7} \mathrm{~m}^{2} / \mathrm{s}$.

To investigate the effect of lowering the diffusion coefficient on the solution, we have run simulations of the bottom section for different values of $D_{s}$. Starting from an homogeneous initial condition, our test case considers a $10 \%$ increase in input concentration. The substrate concentrations at the interface between the bottom and middle sections after 8 hours are shown in Figures 3. Note that the main change of behaviour occurs between $10^{-6} \mathrm{~m}^{2} / \mathrm{s}$ and $10^{-7} \mathrm{~m}^{2} / \mathrm{s}$, when the spatial variations of concentration become sharper and the homogenization effect of diffusion is less important. Therefore we will use $D_{s}=10^{-7} \mathrm{~m}^{2} / \mathrm{s}$ as we are essentially interested in the macroscopic behaviour of the reactor.

\section{Parameter Estimation And Model VERIFICATION}

The computational cost of simulations for this type of model makes it unpractical to fit parameters by minimizing the difference between model outputs and experimental data. Instead, we will use parameter values from [15], that were obtained by fitting a simpler compartment ODE model to experimental data of the reactor that we study here. This simpler model was also based on the 2 reaction model of [16] and it was shown that it is capable of reproducing the 

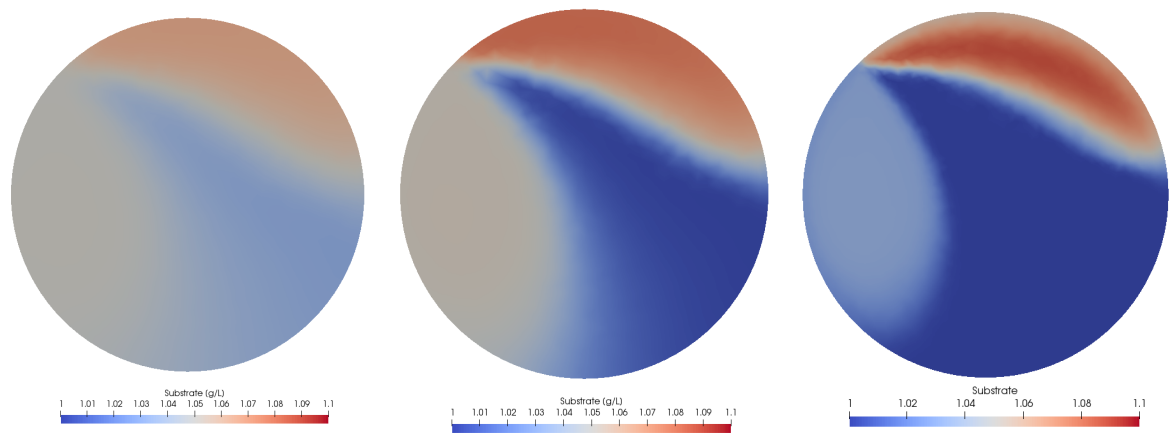

Fig. 3. Bottom-Middle interface at $8 \mathrm{~h}$ after a $10 \%$ change of inflow concentration with mixing flow rate $Q_{m i x}=1000 \mathrm{~L} / \mathrm{h}$ and different diffusion parameters. Left $D=10^{-6}$, Center $D=10^{-7}$, Right $D=10^{-8}$.

input-output behavior of the reactor, so that we will use the same growth function parameters and yield coefficients.

However, this work did not consider density dependent growth functions or biomass death rates and therefore we must estimate these parameters. For the death rates, it is generally accepted that they are small compared to the maximum growth rate and therefore we will take, somewhat arbitrarily, $\tau_{1}=0.1 \cdot \mu_{1}^{\max }$ and $\tau_{2}=0.1 \cdot \mu_{2}^{\max }$.

For the density dependence parameters $\left(c_{1}, c_{2}\right)$, we will use the same experimental data used to fit the model of [15], which contains records of VFA concentrations. There is however no data for $S_{1}$ and therefore to get a first estimate of $c_{1}$, we use the simpler ODE model of [15]. Indeed, this model takes into account the physico-chemical dynamics of the reactor (including $\mathrm{pH}$, alkalinity, strong ions, inorganic carbon and carbon dioxyde) for which experimental data was available and since these variables depend on $S_{1}$, we can assume that the model represents correctly the input-output behaviour of $S_{1}$, especially when the reactor is in a steady state. Therefore, to estimate $c_{1}$, we have run simulations for various values of this parameter and we then take the value for which the steady state of our model is the same as the ODE model [15]. This ODE model considers two perfectly mixed zones in series and the concentrations of the second zone can be interpreted as the output of the reactor. However,

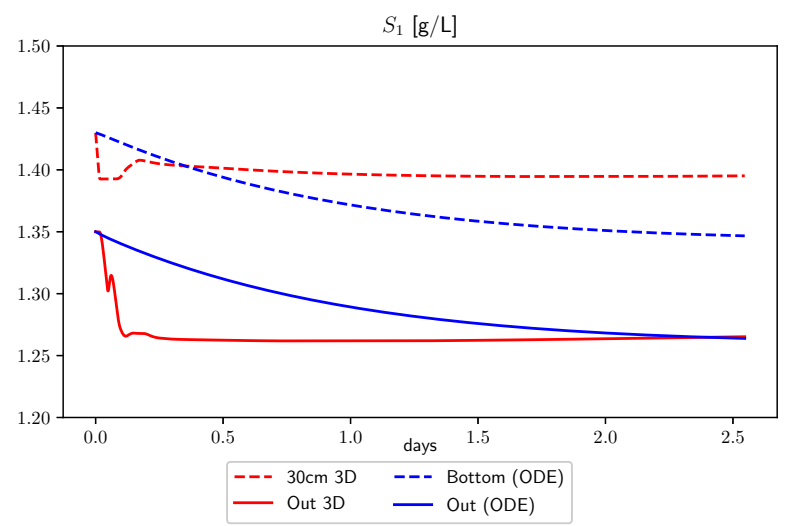

Fig. 4. Concentration of $S_{1}$, for the 3D model at the output and at a height of $30 \mathrm{~cm}$, and for the ODE model of [15] for the first ('Bottom') and second ('Out') zones. it is less obvious which area of the reactor corresponds to the concentrations of the first zone of the ODE model [15] and we therefore focus only on the output of both models. Figure 4 shows the best fit of our 3D model with the ODE model [15].

Now, using experimental data, we can estimate $c_{2}$, the parameter of the second growth function. VFA concentrations were measured from the output but also at several points within the reactor, at heights of 30 and $90 \mathrm{~cm}$ from the bottom. This will allow us to check how well our model can represent the spatial gradient of concentrations within the reactor. Figure 5 illustrates the best fit, as well as the output of the ODE model [15]. The dynamic behaviour is not well reproduced, which is expected as we use data only from a short period of time and a simple 2 reaction model. However, notice that for the data used here, the reactor is essentially in a steady state, which the 3D model is capable of reproducing. More importantly, the 3D model captures well the spatial position where most of the degradation takes place and is also capable of reproducing the vertical variation of concentration better than the ODE model.

Fig. 6 shows the concentrations of $S_{2}$ and $B_{2}$ at steady steady and Fig. 7 shows in detail the concentration of $S_{2}$ in the bottom and top sections. Notice that when a steady state is reached, concentrations are homogeneous in the horizontal

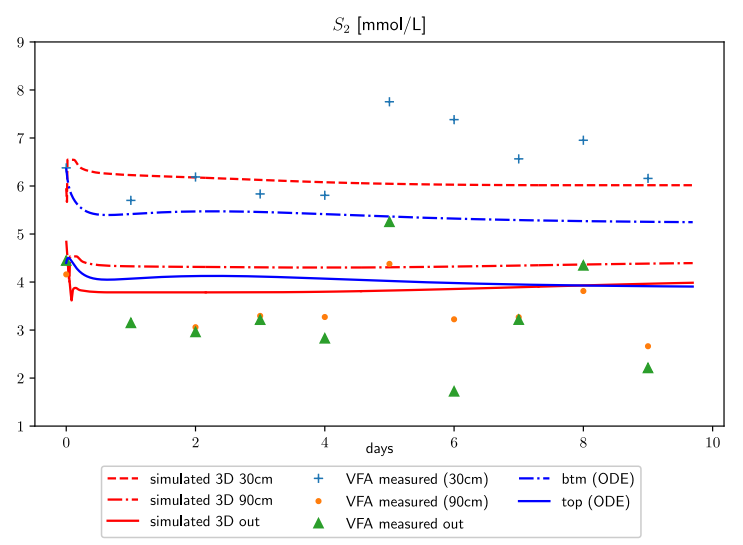

Fig. 5. VFA $\left(S_{2}\right)$ concentrations at different heights within the reactor (30 and $90 \mathrm{~cm}$ from the bottom) and at output, for the 3D model and experimental data. Values of the 2 zone ODE model of [15] are also shown. 


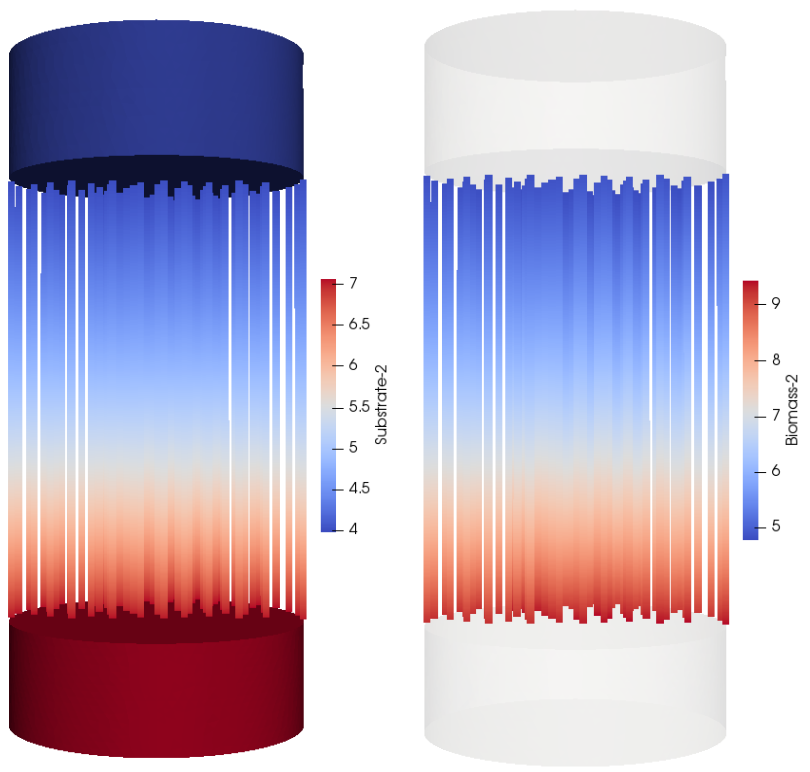

Fig. 6. Distribution of $S_{2}$ (left) and $B_{2}$ (right) at steady state.

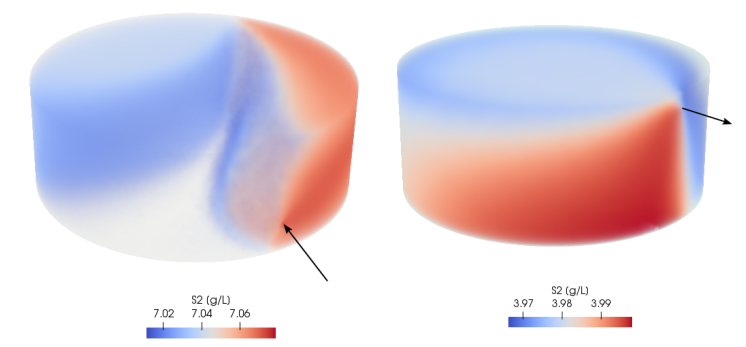

Fig. 7. Distribution of $S_{2}$ in bottom (left) and top (right) sections, arrow indicate input and output.

directions at the scale of the reactor, but nonetheless they are small spatial variations locally.

\section{CONCLUSIONS}

The complexity of the physical and biological processes taking place in bioreactors result in computationally intensive simulations and therefore it is important to develop efficient models, if they are to be used for optimization of bioreactor performance. We develop here a reduced complexity 3D1D model that accurately represents bioreactor geometry and demonstrates the value of simpler models in terms of physical process representation. This model is capable of reproducing the vertical variations of substrate concentration thanks to a density dependent growth rate. This new type of model could be used to better understand the internal dynamics of the reactor and the impact of operational parameters.

\section{ACKNOWLEDGMENT}

Antoine Haddon was supported by FONDECYT grant 1160567, Basal Program CMM-AFB 170001, and doctoral fellowship CONICYT-PFCHA / Doctorado Nacional / 2017 -21170249 from CONICYT-Chile. Powered@ NLHPC: This research was partially supported by the supercomputing infrastructure of the NLHPC (ECM-02).

\section{REFERENCES}

[1] I. Haidar, A. Rapaport, and F. Gérard, "Effects of spatial structure and diffusion on the performances of the chemostat." Mathematical biosciences and engineering: MBE, vol. 8, no. 4, pp. 953-971, 2011.

[2] A. Rapaport, "Some non-intuitive properties of simple extensions of the chemostat model," Ecological complexity, vol. 34, pp. 111-118, 2018.

[3] A. Donoso-Bravo, C. Sadino-Riquelme, D. Gómez, C. Segura, E. Valdebenito, and F. Hansen, "Modelling of an anaerobic plugflow reactor. process analysis and evaluation approaches with non-ideal mixing considerations," Bioresource technology, vol. 260, pp. 95-104, 2018.

[4] M. Terashima, R. Goel, K. Komatsu, H. Yasui, H. Takahashi, Y. Li, and T. Noike, "Cfd simulation of mixing in anaerobic digesters," Bioresource technology, vol. 100, no. 7, pp. 2228-2233, 2009.

[5] M. S. Vesvikar and M. Al-Dahhan, "Flow pattern visualization in a mimic anaerobic digester using cfd," Biotechnology and Bioengineering, vol. 89, no. 6, pp. 719-732, 2005.

[6] M. Meister, D. Winkler, M. Rezavand, and W. Rauch, "Integrating hydrodynamics and biokinetics in wastewater treatment modelling by using smoothed particle hydrodynamics," Computers \& Chemical Engineering, vol. 99, pp. 1-12, 2017.

[7] M. Rezavand, D. Winkler, J. Sappl, L. Seiler, M. Meister, and W. Rauch, "A fully lagrangian computational model for the integration of mixing and biochemical reactions in anaerobic digestion," Computers \& Fluids, vol. 181, pp. 224-235, 2019.

[8] B. Wu, "Integration of mixing, heat transfer, and biochemical reaction kinetics in anaerobic methane fermentation," Biotechnology and bioengineering, vol. 109, no. 11, pp. 2864-2874, 2012.

[9] S. Mu, Y. Zeng, P. Wu, S. Lou, and B. Tartakovsky, "Anaerobic digestion model no. 1-based distributed parameter model of an anaerobic reactor: I. model development," Bioresource technology, vol. 99, no. 9, pp. 3665-3675, 2008.

[10] J. J. Winkin, D. Dochain, and P. Ligarius, "Dynamical analysis of distributed parameter tubular reactors," Automatica, vol. 36, no. 3, pp. 349-361, 2000.

[11] S. Barbier, A. Rapaport, and A. Rousseau, "Modelling of biological decontamination of a water resource in natural environment and related feedback strategies," Journal of Scientific Computing, vol. 68, no. 3, pp. 1267-1280, 2016.

[12] M. Crespo, B. Ivorra, A. M. Ramos, and A. Rapaport, "Modeling and optimization of activated sludge bioreactors for wastewater treatment taking into account spatial inhomogeneities," Journal of Process Control, vol. 54, pp. 118-128, 2017.

[13] _ - "Shape optimization of spatial chemostat models," Electron. $J$ Differential Equations, vol. 2019, no. 84, pp. 1-26, 2019.

[14] M. C. Moya, Mathematical modeling and optimization of bioreactors and liquid crystals. Universidad Complutense de Madrid, 2016.

[15] M. Hmissi, J. Harmand, V. Alcaraz-Gonzalez, and H. Shayeb, "Evaluation of alkalinity spatial distribution in an up-flow fixed bed anaerobic digester," Water Science and Technology, vol. 77, no. 4, pp. 948-959, 2017.

[16] O. Bernard, Z. Hadj-Sadok, D. Dochain, A. Genovesi, and J.-P. Steyer, "Dynamical model development and parameter identification for an anaerobic wastewater treatment process," Biotechnology and bioengineering, vol. 75, no. 4, pp. 424-438, 2001.

[17] C. Lobry and J. Harmand, "A new hypothesis to explain the coexistence of $\mathrm{n}$ species in the presence of a single resource," Comptes Rendus Biologies, vol. 329, no. 1, pp. 40-46, 2006.

[18] L. Halpern, "Artificial boundary conditions for the linear advection diffusion equation," Mathematics of computation, vol. 46, no. 174, pp. $425-438,1986$.

[19] F. Hecht, "New development in freefem++," J. Numer. Math., vol. 20 , no. 3-4, pp. 251-265, 2012. [Online]. Available: https://freefem.org/

[20] O. Pironneau, "On the transport-diffusion algorithm and its applications to the navier-stokes equations," Numerische Mathematik, vol. 38, no. 3, pp. 309-332, 1982.

[21] R. Glowinski and O. Pironneau, "Finite element methods for navierstokes equations," Annual review of fluid mechanics, vol. 24, no. 1, pp. 167-204, 1992.

[22] J. Winkelmann, "Diffusion coefficient of acetic acid into water and ethanol solution," in Diffusion in Gases, Liquids and Electrolytes. Springer, 2017, pp. 1355-1355. 